\title{
Óbitos perinatais evitáveis e ambiente externo ao sistema de assistência: estudo de caso em município da Região Metropolitana do Rio de Janeiro
}

\author{
Avoidable perinatal deaths and the environment \\ outside the health care system: a case study \\ in a city in Greater Metropolitan Rio de Janeiro
}

Maria L. G. Rosa 1

Virginia Alonso Hortale 2

\footnotetext{
1 Consórcio de Acreditação de Sistemas e Serviços de Saúde. Rua São Francisco Xavier 524, Bloco E, Rio de Janeiro, RJ 20559-900, Brasil. 2 Departamento de Administração e Planejamento em Saúde, Escola Nacional de Saúde Pública, Fundação Oswaldo Cruz. Rua Leopoldo Bulhões 1480 Rio de Janeiro, RJ 21041-210, Brasil.
}

\begin{abstract}
This paper focuses on the role of environmental factors external to the health care system in the occurrence of perinatal deaths in maternity hospitals bel onging to the local health system in a city in Greater Metropolitan Rio de Janeiro in 1994. Elements from the political and administrative context that contribute to an understanding of the relationship between failures in health care and structural deficiencies in these maternity hospitals were divided into four groups of variables: distribution of resources, spatial and temporal factors, organizational and managerial features, and action by interest groups. Semi-structured interviews were conducted. The study concluded that poor performance in four groups of variables may have contributed to perinatal mortality: distribution of resources was insufficient to provide quality in health care, especially in private maternity hospitals; there was no formal or informal regi onal or hierarchical organization of obstetric care in the city; Ministry of Heal th guidelines were ignored in all four maternity hospitals, while in three of the hospital s there were no admissions procedures and delivery and fetal follow-up listed in their own rules; and the level of actual participation was low. Key words Maternity Hospitals; Infant Mortality; Perinatal Care; Outcome and Process Assessment; Epidemiology
\end{abstract}

Resumo Este artigo visa compreender a contribuição de fatores do ambi ente externo ao si stema de assi stência na ocorrência de óbitos perinatais em materni dades, que em 1994, compunham a rede de atendimento obstétrico em um muni cípio da região metropol itana do Rio de Janeiro. Dividiram-se os el ementos em quatro grupos de variáveis, para entender a relação entre fal has no atendi mento e deficiências no ambiente externo e institucional izado: repasse de recursos para custei o, fatores geográficos e temporais, características organizacionais e administrati vas e ação/parti ci pação de grupos de interesse. Entrevi stas semi-estruturadas foram real izadas. Os resultados indicaram as seguintes fal has: repasses de recursos para custei o insufi cientes para manter cui dados de qual idade, sobretudo nos casos das maternidades privadas; nenhuma regi onalização ou hi erarquização formal ou informal dos cuidados obstétricos no município; desconheci mento das normas do Ministério da Saúde nas materni dades estudadas e as adotadas em três das quatro maternidades não faziam referência nem aos procedimentos para a admissão, nem ao segui mento do trabalho de parto, nem ao seguimento fetal e o nível de participação não era o efetivamente implementado.

Palavras-chave Maternidades; Mortalidade Infantil; Assistência Perinatal; Avaliação de Processos e Resultados; Epidemiologia 


\section{Introdução}

A reorganização do sistema de saú de brasileiro, iniciada em 1988, com a nova Constituição, culmina, em 1990, com a criação do Sistema Ú nico de Saúde (SUS), que preconiza o acesso e a universalização da atenção à saúde. Sua regulamentação e implementação se dão através das Normas Operacionais Básicas (NOB). A NOB no 1 de 1993 (Brasil, 1993), em vigor no momento da real ização desse estudo, tem como principais pressupostos a descentralização com redefinição de papéis e estabelecimento de novas relações entre as três esferas de governo; o funcionamento regular dos Consel hos de Saúde, paritários e deliberativos; o compartiIhamento do financiamento entre as três esferas do governo e a completa reformulação do modelo assistencial, deslocando-o do modelo centrado no atendimento médico-hospitalar para a atenção integral universal, regionalizada e hierarquizada.

Os serviços hospitalares produzidos, são custeados com base na apresentação da Autorização de Internação Hospitalar (AIH). O teto quantitativo da AlH é proporcional à população e cabe às Secretarias de Saúde e aos ConseIhos de Saúde, seu planejamento e distribuição. Cabe aos Conselhos de Saúde, entre outros, a aprovação dos critérios de programação dos quantitativos de $\mathrm{AlH}$ por ordem de prioridade entre os prestadores públicos, filantrópicos e privados. De acordo com a NOB 93, o município em estudo se enquadra como em gestão parcial, significando que o controle e a avaliação não estavam sob responsabilidade municipal.

Entre os anos de 1994 e 1997, foi realizado um estudo em quatro dimensões e fases, acerca dos óbitos perinatais evitáveis na rede de atendimento obstétrico do SUS, no município em questão. Na primeira fase, os objetivos foram os de conhecer a existência de óbitos que poderiam ser evitados por cuidados adequados não dispensados, e identificar falhas no processo de atendimento que pudessem ter ligação com a ocorrência dos óbitos (publicação inédita). Parte-se de um resultado adverso - morte perinatal potencialmente evitável - como forma de conhecer um problema mais geral: os cuidados inadequados dispensados às mulheres desfavorecidas economicamente. Dezoito casos são classificados como óbitos perinatais potencialmente evitáveis $(56,25 \%$ dos casos considerados para estudo), por terem sido identificadas falhas no processo de atendimento.

As normas usadas como referência são as da American College of Obstetricians and Gyne- cologists (ACOG) e do Ministério da Saúde (MS, 1991), em grande parte coincidentes. Leva-se em consideração a utilização de recursos simples, tanto no que se refere a exames laboratoriais quanto a equipamentos. São identificadas falhas no exame de admissão da mãe à maternidade, dificultando a detecção de uma condição de risco, falhas no acompanhamento da evolução do trabalho, falhas do acompanhamento e monitoramento fetal, um intervalo de tempo grande entre a decisão de fazer uma cesariana e sua efetivação, a não realização de transfusão sangüínea em casos de placenta prévia com hemorragia, a não aspiração de mecônio antes da primeira inspiração do bebê e a utilização inadequada de fórceps. $\mathrm{O}$ atraso ou a não realização de uma transfusão sangüínea, assim como a demora em realizar uma cesariana, são procedimentos que não dependem exclusivamente da decisão médica, mas principalmente, de uma estrutura física e material adequada. As outras deficiências no processo de cuidado têm origem na escolha e/ ou realização de um procedimento inadequado pelo médico.

Duas das fal has observadas na maioria dos prontuários analisados - não identificação de condições de risco e demora em intervir em situações de sofrimento fetal - são sempre descritas em artigos sobre auditorias médicas em perinatalidade (Block, 1990; Ennis \& Vincent, 1990; Nocon \& Coolman, 1987). Verifica-se, através de um questionário respondido por $88,2 \%$ dos obstetras das quatro maternidades do estudo, que a grande maioria desconhecia que a prática cotidiana da maioria desses médicos, se distanciava das normas preconizadas pelo Ministério da Saúde para o acompanhamento do trabalho de parto. Conclui-se que, outros óbitos perinatais potencialmente evitáveis, podem ocorrer nessas maternidades, bastando, para isso, que as condições clínicas adversas das mães e fetos estejam presentes. $\mathrm{Na}$ população atendida por essas maternidades, existia um alto porcentual de mães apresentando fatores de risco para um resultado perinatal adverso, concluindo-se, então, que os óbitos perinatais potencialmente evitáveis, continuariam ocorrendo se não fossem efetivadas medidas para mudar os fatores negativos no processo de trabalho identificados.

Na segunda fase (Rosa \& Hortale, 2000), o objetivo foi o de conhecer a ocorrência de faIhas na estrutura de atendimento. Tentou-se compreender, a partir das deficiências estruturais de cada maternidade, fal has no processo de atendimento obstétrico, identificadas na primeira fase do estudo. Foram incluídos como 
variáveis, os grupos da estrutura física, serviços e equipamentos; da organização de pessoal e das características organizacionais gerais e administrativas. A demora para realizar uma cesariana e de se proceder à transfusão sangüínea, pôde ser explicada por deficiências do grupo de fatores da estrutura física, serviçose equipamentos. As falhas no processo de atendimento obstétrico, sob responsabilidade direta do médico, foram explicadas pelos altos porcentuais de deficiências na organização de pessoal, de deficiências na qualificação profissional e do ambiente profissional de prática. Nas maternidades públicas, foram identificadas maiores oportunidades de melhoria nesse grupo de variáveis. Quanto às características organizacionais e administrativas, constatouse ausência de intervenção, visando compensar as falhas da organização de pessoal nas quatro maternidades, mas nas públicas foi identificado um maior número de falhas relativas ao abastecimento.

Na terceira fase (publicação inédita), buscou-se compreender, à luz da teoria habermasiana, o estado de falência generalizada dessas maternidades.

A pergunta que este artigo visa responder é: Em que medida os grupos de variáveis do ambiente externo e institucionalizado, ajudam a compreender as deficiências no nível organizacional de cada uma das quatro maternidades estudadas (M1, M2, M3, M4), relacionadas à ocorrência de óbitos perinatais potencialmente evitáveis?

\section{Metodologia}

O modelo de análise adotado neste estudo é o de Fottler (1987). Este autor, utilizando o modelo clássico de Donabedian (1992) de avaliação de qualidade: estrutura - processo - resultado, inclui as influências e o impacto do ambiente externo e institucionalizado sobre a estrutura e o processo da organização. O ambiente inclui as regras e as exigências impostas à organização pelos contextos social, jurídico e político, e pelos grupos de interesse (usuários e profissionais). Por sua vez, as variáveis de nível organizacional causam alterações no ambiente externo sob a forma de retroalimentação. A noção de retroalimentação é retirada de Goodman \& Pennings (1980), Lenz (1981) e Scott (1977). Este último, introduz a idéia de se trabalhar sobre o processo, ao invés de se trabalhar sobre as variáveis, como propõe Fottler (1987).

Foram considerados quatro grupos de variáveis: repasse de recursos para custeio, fato- res geográficos e temporais, características organizacionais e administrativas e ação dos grupos de interesse.

Em relação às "variáveis do repasse de recursos para custeio", interessa saber se os valores repassados pelo MS, podem contribuir para as deficiências estruturais em cada maternidade e na rede de serviços de saúde do município. Por não haver sistema de custos à época, nas maternidades do estudo, recorreu-se à uma forma indireta de se atender ao objetivo acima. Compara-se o valor do ressarcimento de uma cesariana feita pelo SUS e pelos seguros privados.

O valor médio da $\mathrm{AlH}$, do município em questão, de julho a dezembro de 1994, de parto normal foi de $\mathrm{R} \$ 118,71$ e o pago por uma cesariana, o equivalente a $\mathrm{R} \$ 195,48$ (DATASUS, 1994). Segundo os valores referenciais de convênios para junho de 1994, negociados com operadoras de seguros e planos de saúde (Golden Cross, Amil, UNIMED, Assim, Bradesco, Sul América e Grupo Ciefas) para planos classe D (os de menor valor), chega-se a R\$ 176,78. São considerados somente os seguintes itens: diárias em enfermarias acima de 2 leitos (três dias), diárias de berçário durante a permanência da mãe (dois dias), taxa de sala de cirurgia porte 3, curativo de umbigo por dois dias, aluguel de bandeja de assepsia, taxa de esterilização, taxa de monitor cardíaco até porte 4, aguIha espinhal (de menor valor), bico de aspirador descartável, clamp umbilical, equipo para soro (1), luva descartável (4), kit obstetrícia de fios agulhados e bandeja de anestesia (Associação dos Hospitais do Rio de Janeiro, 1994). Não foram incluídos medicamentos, roupas para o centro cirúrgico e outros materiais. Note-se que o valor médio repassado pelo SUS, inclui exames laboratoriais e as eventuais complicações, o que este não inclui.

As maternidades públicas e privadas recebem os mesmos valores pelos procedimentos realizados. No caso das maternidades privadas, o montante repassado deve custear pessoal. Para as maternidades públicas, os municípios devem ter orçamento próprio e se responsabilizar pelo pagamento de pessoal.

As variáveis do grupo de "fatores geográficos e temporais", visam mediar a relação entre o acesso ao serviço adequado e os resultados do cuidado. Neste estudo, compara-se o que é definido pela legislação brasileira no que tange ao acesso e à organização dos serviços (hierarquização e organização), ao que é encontrado na pesquisa, buscando-se identificar se há mecanismos de hierarquização informal e seu possível impacto sobre os resultados. 
Quanto às "características organizacionais e administrativas", interessa saber, quais as responsabilidades das três esferas de governo - federal, estadual e municipal no que se refere à normalização das ações, ao monitoramento, controle e avaliação das atividades e dos serviços de saúde.

Para "a ação dos grupos de interesse", faz-se uma análise, ainda que preliminar, das ações dos membros do Conselho Municipal de Saúde e da sua interferência sobre a realidade do atendimento obstétrico e perinatal no município, analisando também, em que medida os dispositivos legais são respeitados, além dos aspectos de participação e paridade. São destacados os principais aspectos referentes à questão dos medicamentos e informações sobre a mortalidade perinatal, visando avaliar a ação e o poder de decisão do Conselho de Saúde. Buscouse, também, avaliar a participação dos usuários no Conselho, a paridade e a escolha dos representantes dos hospitais no Conselho.

Foram utilizadas diversas fontes de informação: listagens fornecidas pelo Departamento de Informática do SUS (DATASUS) com os procedimentos pagos em 1994, para cada uma das quatro maternidades; declarações de nascidos vivos nas quatro maternidades em 1994; declarações de óbitos fetais e neonatais ocorridos nas quatro maternidades em 1994 e até 6 de janeiro de 1995; livros de plantão das maternidades; rotinas e/ ou protocolos clínicos para o atendimento obstétrico e neonatal das maternidades que os possuíam; documentos oficiais do município; legislação brasileira da saúde; diário oficial e lista da Associação de Hospitais do Estado do Rio de Janeiro, com valores negociados com operadoras de seguros e planos de saúde de junho de 1994.

Durante os meses de agosto, setembro e outubro de 1994, foram realizadas entrevistas semi-estruturadas, com informantes-chave: diretores e administradores das maternidades, Secretário Municipal de Saúde, Administrador da Secretaria Municipal de Saúde e membros do Conselho Municipal de Saúde. As entrevistas começaram com os representantes dos usuários no Conselho Municipal de Saúde, para se ter uma noção dos principais problemas identificados por eles e abordá-los com os outros entrevistados. Seu conteúdo foi classificado por tema, ligado ao modelo teórico com a ajuda do programa WordPerfect versão 6.0. Todos os entrevistados deram seus depoimentos após serem informados que o material coletado seria utilizado em tese de doutorado, um documento público.

\section{Resultados}

A Tabela 1, apresenta algumas características da estrutura e produção das quatro maternidades. Na época do estudo, nenhuma das quatro maternidades tinha Centro de Terapia Intensiva (CTI) de adulto ou neonatal.

\section{Repasses de recursos para custeio}

A fala dos técnicos do MS mostrou, de um lado, que consideravam que os recursos repassados eram insuficientes para custear cuidados de qualidade, e de outro, que havia suspeita de fraude:

“Sim, o governo paga muito pouco, mas seas maternidades privadas continuam a funcionar... é porqueisso vale a pena. Elas sempre encontraram formas de chegar ao lucro. ...Nós tentamos, de toda forma, coibir a fraude, mas ela ainda existe" (coordenadora de operações e controle de serviços).

“Bem, com os recursos do Ministério da Saúde, que se reduzem de mais a mais, não há forma de se fazer mais. Nós tentamos corrigir os valores dentro do orçamento. ...si m o governo paga muito pouco mas seas maternidades continuam a funcionar...é porque vale a pena" (coordenador de desenvolvimento e controle de serviços).

Comparando-se o número de partos ressarcidos pelo MS ao número de declarações de nascido vivo, excluídos os gemelares, acrescidos às declarações de óbito de natimortos, observou-se que o número de partos ressarcidos não foi maior em nenhuma das quatro maternidades. No caso das duas maternidades públicas, o número de partos realizados foi maior que o ressarcido, mostrando haver negligência administrativa ou incompetência no preenchimento das faturas. $\mathrm{O}$ argumento de fraude que acompanha o discurso das autoridades não se confirmou (Rosa \& Hortale, 2000).

\section{Fatores geográficos e temporais}

O artigo no 198 da Constituição Federal do Brasil (Brasil, 1988), estipula que as ações e os serviços de saúde integram uma rede regionalizada e hierarquizada, e constituem um sistema único. A NOB no 01 de 1993, reforça essa diretriz quando afirma que a regionalização deve ser entendida como uma articulação e mobilização municipal, que leve em consideração características geográficas, fluxo de demanda, perfil epidemiológico, oferta de serviços e, acima de tudo, a vontade política expressa pelos diversos municípios de se consorciar ou esta- 
Estrutura e produção de quatro maternidades em município da Região Metropolitana. Rio de Janeiro, 1994.

\begin{tabular}{lcccc}
\hline $\begin{array}{l}\text { Características } \\
\text { Tipo }\end{array}$ & $\begin{array}{c}\text { M1 pública } \\
\text { (municipal) }\end{array}$ & $\begin{array}{c}\text { M2 pública } \\
\text { (municipal) }\end{array}$ & M3 conveniada & M4 conveniada \\
\hline Porte & médio & pequeno & médio & pequeno \\
Número de médicos & 63 & 49 & 16 & 6 \\
Número de leitos & 35 & 32 & 93 & 26 \\
Mesas de parto & 2 & 2 & 62,03 & 1 \\
\% do total de partos & 18,64 & 12,26 & 25,83 & 7,07 \\
\% de cesarianas & 11,36 & 12,26 & 27,08
\end{tabular}

belecer qualquer outra relação de caráter cooperativo.

Com base nas entrevistas realizadas com os administradores das quatro maternidades e com o Secretário Municipal de Saúde, verificou-se que não existe hierarquização nem regionalização da rede de atendimento obstétrico na zona metropolitana do Rio e Janeiro. No município em estudo, a situação parece ainda mais caótica. O hospital, que é referência informal para as três maternidades é, sem dúvida, pelos resultados apresentados em um dos artigos (Rosa \& Hortale, 2000), o que tem os problemas estruturais mais sérios.

O baixo peso ao nascer foi utilizado como variável proxi de risco. A Tabela 2, apresenta a distribuição dos nascidos vivos segundo a categoria de peso, nas quatro maternidades.

$\mathrm{Na} \mathrm{M} 1,14,51 \%$ dos bebês nasceram com peso inferior a 2.500 g e na $M 2,12,32 \%$. Já nas duas maternidades privadas, o porcentual foi de 4,45 e 4,68. O livro de ocorrências mostrou transferência de gestantes da $M 2$ para a $M 1$, principalmente nos fins de semana. Na M3, o diretor assegurou que a maternidade é nível 1 , onde são aceitas gestantes de baixo risco. As gestantes de alto risco são aceitas em último caso. Na M 4, o diretor informou que a maternidade não aceita gestantes que não sejam de baixo risco e que os partos de alto risco, são realizados somente quando não há tempo hábil para a transferência.

\section{Características organizacionais e administrativas}

As taxas de mortalidade materna e perinatal, no município e nas maternidades do SUS, não eram conhecidas por nenhum dos entrevistados. Entende-se que essas informações deveriam ser base para as ações de saúde, auxilian- do na tomada de decisão de administradores e membros do Conselho de Saúde. Não existiam, segundo o Secretário Municipal de Saúde ou os membros do Conselho Municipal de Saúde, propostas visando a formação dos recursos humanos para que fossem corrigidas as deficiências técnicas dos profissionais (Rosa \& Hortale, 2000).

As normas do MS, muito próximas das adotadas no estudo (AAP \& ACOG, 1992), não eram conhecidas em nenhuma das maternidades. (Rosa \& Hortale, 2000).

\section{Ação dos grupos de interesse}

O Consel ho Municipal de Saúde do município estudado, foi regulamentado em 1992, com caráter permanente e deliberativo. Foram entrevistados cinco participantes do Conselho: dois representantes dos usuários, dois representantes dos profissionais de saúde e um representante da administração municipal.

Na questão dos medicamentos, ficou subentendida a pequena participação do ConseIho na sua deliberação: "A lei que regulamenta o funcionamento do SUS, no município, atribui à administração central da Prefeitura enão à Secretaria Municipal deSaúde, a atribuição das compras..." (representante dos usuários).

Quanto às informações sobre a mortalidade perinatal, as entrevistas indicaram o baixo nível de informação existente entre os representantes do Conselho:

“Essa questão nunca foi abordada no ConseIho" (representante dos profissionais de saúde).

“Na realidade, nós não temos nenhuma explicação através da rede oficial...nós sabemos quea qual idadedo atendimento não éboa. Mas como criar uma consciência, como fiscalizar, exigir ? É tudo um processo e ainda estamos longe..." (representante dos usuários). 
Tabela 2

Distribuição porcentual dos nascidos vivos, segundo a categoria de peso em gramas e maternidade. Município da Região Metropolitana do Rio de Janeiro, 1994.

\begin{tabular}{lrrrr}
\hline \multirow{2}{*}{ Categoria de peso } & \multicolumn{4}{c}{ Maternidade } \\
& M1 (\%) & M2 (\%) & M3 (\%) & M4 (\%) \\
\hline$<2.500 \mathrm{~g}$ & 14,51 & 12,32 & 4,45 & 4,68 \\
$2.500-4.500 \mathrm{~g}$ & 85,05 & 87,13 & 95,03 & 93,76 \\
$>4.500 \mathrm{~g}$ & 0,37 & 0,44 & 0,52 & 1,56 \\
Sem informação & 0,07 & 0,11 & 0,00 & 0,00 \\
Total & 100,00 & 100,00 & 100,00 & 100,00 \\
\hline
\end{tabular}

Quanto ao poder de decisão do Conselho de Saúde, também ficou evidente sua pouca capacidade de controle e pressão:

“Legalmente, o Consel ho tem poderes, mas como todas as coisas, émuito político. Quer dizer, énecessariamente político. E a correlação de forças émuito difícil para o usuário..." (representante dos usuários).

As respostas dos provedores e dos usuários divergem quando se fala de paridade. Para o representante dos provedores “...o Consel ho é totalmente paritário". Entretanto, para um dos representantes dos usuários “...a pari dade não é respeitada...a relação deveria ser de 16/16, mas na realidade está entre 12/20...".

Na mesma linha de raciocínio do usuário anterior, “...não podemos contar com o representante da Faculdade do município, nem com o representante do Poder Legisl ativo do município que conseguiram ficar no lugar do usuário..." (representante dos usuários).

Quanto à escolha dos representantes dos hospitais públicos, as entrevistas explicitaram a dificuldade em eleger representantes, o que, se fosse mais fácil, ampliaria a partici pação:

“...nós não queremos que os diretores das unidades de saúde sejam representantes das unidades no Consel ho. Queremos que o repre sentanteseja el eito..." (representante dos usuários).

“...o representante de um hospital, por exemplo, éo diretor. Elenão abrea boca, não diz nada sobre a maternidade, se acontece al go de grave ele não menciona..." (representante dos profissionais de saúde). “...Sim, para não perder o seu cargo..."(representante dos usuários).

O que se observou é que, o Conselho Municipal de Saúde conhecia superficialmente o que ocorria nos serviços, e seu poder de intervenção era limitado (como se viu no caso dos medicamentos). A pouca participação dos representantes das maternidades públicas no
Conselho também ficou evidente. Na verdade, o Consel ho não cumpria a contento suas obrigações legais na formulação de políticas e avaliação dos serviços prestados. Sua não ação foi um fator que contribuiu para o quadro de faIhas em nível das maternidades.

\section{Discussão}

Quanto às variáveis do grupo repasse de recursos para custeio, viu-se que os val ores transferidos pelo SUS equivaliam aos repassados pelos convênios com operadoras de seguros e planos de saúde para a classe de menor valor (D), excluindo pessoal, medicamentos, roupas de centro cirúrgico e outros materiais. Não considerava os exames diagnósticos nem as complicações. O argumento de fraude, aventado em entrevista com técnicos do MS, não se confirmou. Os resultados mostraram que a rede privada recebia do SUS menos recursos que a rede pública, pois nesta, o pagamento de pessoal era feito com recursos do orçamento municipal. Os resultados indicaram que, principalmente na rede privada, o repasse insuficiente ajudava a explicar as fal has na estrutura das maternidades estudadas.

No que tange às variáveis grupo fatores geográficos e temporais, as entrevistas confirmaram que não existia regionalização ou hierarquização formal de cuidados obstétricos na Região Metropolitana do Rio de Janeiro. A situação no município em estudo se caracterizava pelo seguinte: a maternidade de referência informal das três maternidades, era a M1. Essa maternidade era, sem dúvida, aquela que tinha os problemas estruturais mais sérios, embora recebesse as gestantes de risco. $O$ estudo indicou, que as maternidades privadas continuavam atendendo a clientela do SUS, com prejuízos visíveis na qualidade dos cuidados ofertados, conforme descrito na introdução desse artigo. A hierarquização informal, funcionava, na verdade, como uma válvula de defesa das três maternidades. As quatros maternidades não estavam estruturadas para receber gestantes de risco. A saúde é um dever do Estado, o governo municipal encarna o Estado, logo a M 1, principal maternidade municipal, deveria estar estruturada para receber as gestantes de risco, pelo menos de médio risco, do município. Mesmo não estando estruturada para tal, era esse o papel que a M 1, de forma compulsória, acabava desempenhando.

Em relação às características organizacionais e administrativas, sabe-se que as principais atribuições legais dos três níveis adminis- 
trativos são, para o nível central (Ministério da Saúde): as de monitorar, controlar eavaliar as ações e os serviços de saúde, respeitar as compe tências dos estados e municípios. Para o nível estadual (Secretaria Estadual de Saúde): estabelecer normas de caráter complementar, para o controle e avaliação das ações e serviços de saúde; controlar efiscalizar os procedimentos dos serviços privados de saúde. E para o nível municipal (Secretaria Municipal de Saúde) são definidos: planejar, organizar, controlar eavaliar as ações eserviços de saúde; gerenciar eexecutar os serviços públi cos de saúde; participar no planej amento, programação e organização da rede regi onalizada e hierarquizada do SUS, em articulação com o nível estadual; controlar efiscalizar os procedimentos dos serviços privados de saúde (Brasil, 1990a). As entrevistas com os membros do Conselho Municipal de Saúde, indicaram que existiam diferenças entre o realizado e o previsto na Constituição. Nem as ações para tornar conhecidas as normas estabelecidas na área materno-infantil, nem aquelas de monitoramento, controle e avaliação das atividades e dos serviços de saúde eram implementadas pelos administradores dos três níveis administrativos (Federal, Estadual, Municipal).

Quanto à padronização dos procedimentos nas áreas materno-infantil do MS, foram publicadas em 1991, as normas para o atendimento materno e perinatal. Nesse documento é dito que a padronização das ações (maternas e perinatais) é, sem dúvida alguma, uma atividade estratégica no processo de organização do SUS e condição para desenvolver ações de impacto na saúde da população. Assim, essa padronização deve ser prioritária (MS, 1991). É importante lembrar, que as normas para a atenção materno-infantil do Ministério da Saúde, mais especificamente as de monitoramento do parto, não eram conhecidas em nenhuma das maternidades estudadas. Por outro lado, as normas adotadas em três das quatro maternidades, não faziam referência nem aos procedimentos para a admissão, nem ao seguimento do trabalho de parto, nem ao seguimento fetal.

Em relação aos grupos de interesse, as entrevistas indicaram que a ação pretendida pela Lei no 8.142 (Brasil, 1990b), não é a efetivamente implementada, devido a problemas de ordem política e cultural.

\section{Considerações finais}

Os quatro grupos de variáveis do ambiente externo considerados no estudo, são objeto da
Lei no 8.080, de 19 de setembro de 1990, e da NOB 93. Nos quatro casos, foram identificadas deficiências que podem estar ligadas com as deficiências no processo e na estrutura da assistência ao parto e ao neonato, e por conseguinte, relacionadas à ocorrência de óbitos perinatais potencialmente evitáveis. Não foi previsto analisar a relação entre os grupos de variáveis no modelo adotado no estudo, no entanto, cabem algumas considerações a esse propósito.

O estudo indicou que o baixo teto financeiro destinado ao custeio das atividades das quatro maternidades, influenciou negativamente na qualidade dos serviços oferecidos. No entanto, esse efeito negativo poderia ser minimizado por variáveis dos demais grupos, o que não aconteceu.

A regionalização e a hierarquização do sistema são um dos pilares do SUS. O que se viu, foi a total ausência de regionalização e hierarquização formais, havendo uma hierarquização informal e perversa, na medida em que as gestantes de risco não eram transferidas para sua proteção e sim para proteção das maternidades.

A Lei no 8.080 , dispõe que é de competência da direção nacional do SUS: monitorar, controlar e avalias as ações e os serviços de saúde; do Estado de estabelecer normas de caráter suplementar e do Município: de controlar e avaliar as ações e serviço de saúde. O MS publicou em 1991, o manual Assistência Institucional ao Parto, ao Puerpério e ao Recém-nascido (MS, 1991:5) que afirma que, "a padronização das ações na área de assistência ao parto e ao neonato, é uma atividade estratégica no processo de organização do SUS e uma pré-condição para que as ações tenham um impacto positivo na saúde da população". O manual diz também que, o MS, responsável por traçar a política do setor, envida esforços para padronizar e divulgar as normas. No entanto, o estudo mostrou que as três instâncias falharam em disseminar as normas do MS e em monitorar e avaliar os serviços de atenção materno-infantil. Nem mesmo os coeficientes de mortalidade materna e perinatal eram conhecidos.

O estudo descreveu, a não ação do ConseIho de Saúde e apontou para a existência de uma paridade questionável, limitando o poder de participação dos usuários. Uma das características da participação, de acordo com Borja (1984:17), é a defesa dos chamados “direitos ampliados" dos cidadãos ao ambiente externo, à informação, à saúde, entre outros. A possibilidade do exercício efetivo dos seus direitos perante a administração pública, bem como sua 
organização, requerem âmbitos territoriais reduzidos e interlocutores muito próximos. Tal é o caso dos Conselhos Municipais de Saúde, em municípios de médio porte, que têm ConseIhos Comunitários de Saúde representando os usuários, organizado por bairros, como no município em estudo.

No plano da administração pública, a essência da participação, para Jacobi (1991), reside na possibilidade que os usuários dos serviços têm de opinar e também de participar ativamente na implementação e gestão dos serviços públicos, influenciando o processo decisório.

A implementação das propostas de participação tem esbarrado num somatório de fatores, com destaque para "a falta de agilidade nas decisões, o problemático compromisso do corpo de funcionários e, principal mente, a ausência de critérios de representação e canais admi nistrati vos que garantam o suporte insti tucional à interação com os grupos mais organizados e com os movimentos populares" (Jacobi, 1991:37). Como conseqüência, essas situações tornam os canais de comunicação escassos e a participação frágil.

Neste estudo, algumas das teses expressas pelos autores, acima são confirmadas: a de que o nível de participação pretendido pela Lei $n^{\circ}$ 8.142, não foi efetivamente implementado, devido a problemas de duas ordens: política e cultural. De um lado, pela fraqueza da sociedade civil organizada, predominando o clientelismo e o paternalismo. De outro, pelo arranjo político-institucional estabelecido. A composição do Conselho Municipal de Saúde no muni- cípio em questão, foi regulamentada pela Câmara de Vereadores, de caráter conservador. Assim, votam como usuários, representantes de "Clube de Serviços" (Rotary e Lions Club), um representante da própria Câmara de Vereadores, um da Faculdade do município, um da Ordem de Advogados e um da Associação Médica. O acesso ao SUS é universal, no entanto, seus usuários no país como um todo e no município em estudo, são trabalhadores de baixa renda, e os membros das instituições citadas, além de não serem usuários dessa rede, têm interesses diferentes dos primeiros. Assim, os representantes dessas entidades, votam tradicionalmente contra as propostas apresentadas pelos representantes dos efetivos usuários da rede do SUS, os membros do Conselho Comunitário de Saúde.

É certo que não há como relacionar a composição real do Conselho Municipal de Saúde à sua não ação. No entanto, considerou-se importante mostrar que, no contexto do município estudado, a participação de usuários, refletida na paridade, enquanto forma de expressão do interesse público, elemento essencial da política de descentralização, outro pilar de afirmação do SUS, era bastante questionável.

Conclui-se que, esse conjunto de ocorrências, de omissões das instâncias do ambiente externo e institucionalizado, contribuiu para as fal has na estrutura e processo de cuidado relacionados à ocorrência dos óbitos perinatais potencialmente evitáveis, ocorridos nas maternidades que compunham a rede SUS no município estudado.

\section{Referências}

AAP (American Academy of Pediatrics)/ ACOG (American College of Obstetricians and Gynecologists), 1992. Guidelines for Perinatal Care. 3rd Ed. Washington, DC: AAP/ACOG.

ASSOCIAÇÃO DOS HOSPITAIS DO RIO DE JANEIRO, 1994. Valores Referenciais de Convênio. Pesquisa de Preços de Materiais Descartáveis. Circular 25. Rio de Janeiro: Associação dos Hospitais do Rio de Janeiro.

BLOCK, B. S., 1990. Evaluating the quality of perinatal health care. Americam Journal of Perinat, 7:146153 
BRASIL, 1990a. Lei 8.080, de 19 de setembro de 1990. Dispõe sobre as condições para a promoção, proteção e recuperação da saúde, a organização e o funcionamento dos serviços correspondentes e dá outras providências. Brasília: Diário Oficial da República Federativa do Brasil, no 182, 20 set. pp. 18055-18059.

BRASIL, 1990b. Lei 8.142, de 28 de dezembro de 1990. Dispõe sobre a participação da comunidade na gestão do Sistema Único de Saúde - SUS e sobre as transferências intergovernamentais de recursos financeiros na área da saúde e dá outras providências. Brasília: Diário Oficial da República Federativa do Brasil, no 249, 31 dez. pp. 2569425695.

BRASI L, 1993. Portaria no 545: Estabel ece Normas e Procedimentos Reguladores do Processo de De scentral ização da Gestão das Ações e Serviços de Saúde, Através da Norma Operacional Básica, SUS 01/93. Brasília: Ministério da Saúde.

BORJA, J., 1984. Descentralización: Una cuestión de metodo. Revista Mexicana de Sociologia, 46:5-33.

DATASUS (Departamento de Informática do SUS), 1994. Dados da Autorização de Internações Hospitalares, 1994. বhttp:// www.tabnet.datasus.gov.br>.

DONABEDIAN, A., 1992. The role of outcomes in quality asessment assurance. Quality Review Bulletin, 18:356-360.

ENNIS, M. \& VINCENT, C. A., 1990. Obstetric accidents: A review of 64 cases. BMJ, 300:1365-1367.

FOTTLER, M. D., 1987. Health care organisational performance: Present and future research. Journal of Management, 13:367-391.
GOODMAN, P. S. \& PENNINGS, J. M., 1980. Critical issues in assessing organizational effectiveness. In: Organizational Assessment. Perspectives on the Measurement of Organizational Behavior and the Quality of Life (E. E. Lawler III, ed.), New York: John Wiley \& Sons.

JACOBI, P., 1991. Os municípios e a participação. Revista deAdministração Municipal, 38:32-38.

KIRKUP, B. \& WELCH, G., 1990. Normal bur dead: Perinatal mortality in non-malformed babies of birth-weight $2.5 \mathrm{~kg}$ and over in the Northen Region in 1983. British Journal of Obstetrics and Gynecology, 97:381-392.

LENZ, R. T., 1981. 'Determinants' of organizational performance: An interdisciplinary review. Strategic Manangement Journal, 2:131-154.

MS (Ministério da Saúde), 1991. Assistência Institucional ao Parto, ao Puerpério eao Recém-nascido. Brasília: MS.

NOCON, J. J. \& COLMAN, D. A., 1987. Periantal malpractice: Risk and prevention. Journal of Reproductive Medicine, 32:83-90.

ROSA, M. L. G. \& HORTALE, V. A., 2000. Óbitos perinatais evitáveis e a estrutura de atendimento obstétrico na rede pública: Estudo de caso de um Município da Região Metropolitana do Rio de Janeiro. Cadernos deSaúde Pública, 16:773-783.

SCOTT, R. W., 1977. Effectiveness of organizational studies. In: New Perspectives on Organizational Effectiveness (P. S. Goodman \& J. M. Pennings, ed.), New York: Jossey Bass Publishers.

Recebido em 12 de maio de 1999

Versão final reapresentada em 25 de julho de 2001

Aprovado em 8 de outubro de 2001 